1 Hacettepe Journal of Mathematics and Statistics

$\bigcap$ Volume 43 (6) (2014), 963-969

\title{
Common fixed and coincidence point theorems for maps in Menger space with Hadzic type $\mathrm{t}$ - norm
}

\author{
K.P.R.Rao* ${ }^{*}$ K.Bhanu Lakshmi ${ }^{\dagger}$ and N.Srinivasa Rao ${ }^{\ddagger}$
}

\begin{abstract}
In this paper, we obtain a unique common fixed point theorem for two weakly compatible mappings in a Menger space and also obtain a common coincidence point theorem for two hybrid pairs of mappings.

2000 AMS Classification: 47H10, 54H25.
\end{abstract}

Keywords: Menger space, Hadzic type t-norm, weakly compatible mappings.

Received 30:01:2013 : Accepted 18:11:2013 Doi : 10.15672/HJMS.2014437530

\section{Introduction and preliminaries}

In 1942, Menger [6] introduced the notion of a statistical metric space as a generalization of a metric space $(M, d)$ in which the distance $d(x, y),(x, y \in M)$ between $x$ and $y$ is replaced by a distribution function $F_{x, y}$. Schweizer and Sklar [9] studied this concept and established some fundamental results on this space. First, we give some known preliminaries.

1.1. Definition. . A mapping $F: R \rightarrow[0,1]$ is said to be a distribution function if

(i) $F$ is non-decreasing,

(ii) $F$ is left continuous,

(iii) $\inf _{x \in R} F(x)=0$ and $\sup _{x \in R} F(x)=1$.

We denote the set of all distribution functions by $\mathbb{D}$.

${ }^{*}$ Department of Mathematics, Acharya Nagarjuna University, Nagarjuna Nagar -521 510, A.P., INDIA.

Email: kprrao2004@yahoo.com

${ }^{\dagger}$ Department of Science and Humanities, Lakireddy Balireddy College of Engineering, Mylavaram -522 230, A.P., INDIA.

Email:bhanulaks@gmail.com

${ }^{\ddagger}$ Department of Science and Humanities, Vignan University,Vadlamudi-522 213, Guntur Dt., A.P., INDIA.

Email:srinivasunimmala@yahoo.co.in 
1.2. Definition. ([9]). A probabilistic metric space is an ordered pair $(M, F)$, where $M$ is a non empty set and $F$ is a function defined on $M \times M$ to $\mathbb{D}$ which satisfies the following conditions: For $x, y, z \in M$,

(i) $F_{x, y}(0)=0$

(ii) $F_{x, y}(s)=1$ for all $s>0$ if and only if $x=y$,

(iii) $F_{x, y}(s)=F_{y, x}(s)$ for all $s \in R$ and

(iv) $F_{x, y}\left(s_{1}\right)=1$ and $F_{y, z}\left(s_{2}\right)=1$ for all $s_{1}, s_{2}>0$ imply $F_{x, z}\left(s_{1}+s_{2}\right)=1$.

1.3. Definition. ([9]). A function $t:[0,1] \times[0,1] \rightarrow[0,1]$ is said to be a triangular norm or $t$ - norm if it satisfies the following conditions: For $a, b, c, d \in[0,1]$,

(i) $t(a, 1)=a$

(ii) $t(a, b)=t(b, a)$

(iii) $t(c, d) \geq t(a, b)$ if $c \geq a$ and $d \geq b$,

(iv) $t(t(a, b), c)=t(a, t(b, c))$.

1.4. Definition. ([9]). Let $M$ be a nonempty set, ' $t$ ' is a $t$ - norm and $F: M \times M \rightarrow \mathbb{D}$ satisfy:

(i) $F_{x, y}(0)=0$ for all $x, y \in M$,

(ii) $F_{x, y}(s)=1$ for all $s>0$ if and only if $x=y$,

(iii) $F_{x, y}(s)=F_{y, x}(s)$ for all $s \in R$ and

(iv) $F_{x, y}(u+v) \geq t\left(F_{x, z}(u), F_{z, y}(v)\right)$ for all $u, v \geq 0$ and $x, y, z \in M$.

Then the triplet $(M, F, t)$ is called a Menger space.

1.5. Remark. If $(M, d)$ is a metric space then ' $d$ ' induces a mapping $F: M \times M \rightarrow \mathbb{D}$ , where $F$ is defined by $F_{p, q}(x)=H(x-d(p, q))$, where $H(x)=\left\{\begin{array}{ll}0 & \text { if } x \leq 0, \\ 1 & \text { if } x>0\end{array}\right.$ is the Heaviside function.

Further, if $t:[0,1] \times[0,1] \rightarrow[0,1]$ is defined by $t(a, b)=\min \{a, b\}$, then $(M, F, t)$ is a Menger space. It is complete if the metric space $(M, d)$ is complete.

1.6. Definition. ([9]). Let $(M, F, t)$ be a Menger space. Let $x \in M$. For $\epsilon>0$ and $0<\lambda<1$, the $(\epsilon, \lambda)$ - neighbourhood of $x$ is defined as $N_{x}(\epsilon, \lambda)=\left\{y \in M: F_{x, y}(\epsilon)>1-\lambda\right\}$.

The topology induced by the family $\left\{N_{p}(\epsilon, \lambda): p \in M, \epsilon>0,0<\lambda<1\right\}$ is known as the $(\epsilon, \lambda)$ - topology.

1.7. Proposition. ([9]). If $t$ is continuous then $(\epsilon, \lambda)$ - topology is a Hausdorff topology on $M$.

1.8. Definition. ([9]). Let $(M, F, t)$ be a Menger space. A sequence $\left\{x_{n}\right\}$ in $M$ converges to $x \in M$, if for any $\epsilon>0$ and $0<\lambda<1$, there exists a positive integer $N=N(\epsilon, \lambda)$ such that $F_{x_{n}, x}(\epsilon)>1-\lambda$ for all $n \geq N$. A sequence $\left\{x_{n}\right\}$ in $(M, F, t)$ is said to be Cauchy sequence in $M$ if for $\epsilon>0$ and $0<\lambda<1$, there exists a positive integer $N=N(\epsilon, \lambda)$ such that $F_{x_{n}, x_{m}}(\epsilon)>1-\lambda$ for all $n, m \geq N$. A Menger space $(M, F, t)$, where $t$ is continuous, is said to be complete if every Cauchy sequence in $M$ is convergent in $(\epsilon, \lambda)$ - topology.

In 1972, Sehgal and Reid [10] introduced the notion of contraction mapping on a probabilistic metric space and proved fixed point theorems for such mappings. 
1.9. Definition. ([10]). Let $(M, F, t)$ be a Menger space. A map $T: M \rightarrow M$ is said to be a contraction mapping if there exists a constant $0<p<1$ such that $F_{T x, T y}(s) \geq F_{x, y}\left(\frac{s}{p}\right)$ for each $x, y \in M$ and for all $s>0$.

1.10. Theorem. ([10]). Let $(M, F, t)$ be a complete Menger space, where ' $t$ ' is a continuous function satisfying $t(x, x) \geq x$ for each $x \in[0,1]$. If $T: M \rightarrow M$ is a contraction mapping then there is a unique $p \in M$ such that $T p=p$. Moreover $T^{n} q \rightarrow p$ for each $q \in M$.

In 1978, Hadzic [4] introduced a class $\mathcal{F}$ of $t$ - norms $t \neq t_{\min }$, for which every contraction in a complete Menger space $(M, F, t)$ has a fixed point.

1.11. Definition. ([4]). We say that the $t$ - norm $t$ is of Hadzic - type and we write $t \in \mathcal{F}$ if the family $\left\{t^{n}\right\}_{n \in N}$ of it's iterates defined, for each $x \in[0,1]$ by $t^{0}(x)=1$ and $t^{n+1}(x)=t\left(t^{n}(x), x\right)$ for all $n \geq 0$ is equicontinuous at $x=1$.

i.e., for each $\epsilon \in(0,1)$, there exists $\delta \in(0,1)$ such that $x>1-\delta$ implies $t^{n} x>1-\epsilon$ for all $n \geq 1$.

1.12. Theorem. ([4]). Let $(M, F, t)$ be a complete Menger space, where ' $t$ ' is a continuous $t$ - norm of Hadzic type. If $T: M \rightarrow M$ is a contraction mapping then there is a unique $p \in M$ such that $T p=p$. Moreover $T^{n} q \rightarrow p$ for each $q \in M$.

Recently Choudhury and Das [1], proved the following

1.13. Theorem. ([1]). Let $\left(M, F, t_{M}\right)$ be a complete Menger space with continuous $t$-norm $t_{M}$ given by $t_{M}(a, b)=\min \{a, b\}$ and $f: M \rightarrow M$ be satisfying $F_{f x, f y}(\varphi(s)) \geq$ $F_{x, y}\left(\varphi\left(\frac{s}{c}\right)\right)$ for all $x, y \in M$ and for $s \geq 0$, where $0<c<1$ and $\varphi: R \rightarrow R^{+}$satisfies

(i) $\varphi(t)=0$ iff $t=0$,

(ii) $\varphi(t)$ is increasing and $\varphi(t) \rightarrow \infty$ as $t \rightarrow \infty$,

(iii) $\varphi$ is left continuous on $(0, \infty)$,

(iv) $\varphi$ is continuous at 0 .

Then $\mathrm{f}$ has a unique fixed point in $M$.

Later several authors obtained fixed point theorems in Menger spaces using an altering distance function,for example refer [2],[3],[7]etc.

Sastry et.al. [8], defined altering function of type (S) as follows :

1.14. Definition. ([8]) A function $\varphi: R^{+} \rightarrow R^{+}$is said to be an altering distance function of type $(\mathrm{S})$ if it satisfies

(i) $\varphi(t)=0$ iff $t=0$,

(ii) $\varphi(t) \rightarrow \infty$ as $t \rightarrow \infty$,

(iii) $\varphi$ is continuous at 0 .

1.15. Lemma. ([8]) Let $(M, F, t)$ be a Menger space with a continuous Hadzic type $t$ norm , $0<c<1$ and $\varphi$ be an altering distance function of type (S). Suppose $\left\{x_{n}\right\}_{n=0}^{\infty}$ is a sequence in $M$ such that for any $r>0, F_{x_{n}, x_{n+1}}(\varphi(r)) \geq F_{x_{0}, x_{1}}\left(\varphi\left(\frac{r}{c^{n}}\right)\right)$. Then $\left\{x_{n}\right\}$ is a Cauchy sequence.

1.16. Theorem. ([8]) Let $(M, F, t)$ be a complete Menger space with a continuous Hadzic type $t$ - norm ' $t$ ' and $\varphi$ be an altering distance function of type (S), $P: M \rightarrow M$ be satisfying $F_{P x, P y}(\varphi(s)) \geq F_{x, y}\left(\varphi\left(\frac{s}{c}\right)\right)$ for all $x, y \in M$ and for $s>0$ and $0<c<1$. Then $\mathrm{P}$ has a unique fixed point $z \in M$. Moreover, $P^{n} x \rightarrow z$ for each $x \in M$.

1.17. Definition. ([5]) A pair of self mappings is called weakly compatible if they commute at their coincidence points.

In this paper, we extend Theorem 1.16 for two pairs of weakly compatible mappings. 


\section{Main results}

2.1. Theorem. Let $(M, F, t)$ be a Menger space with continuous Hadzic type $t$-norm ' $t$ ' and $\varphi$ be an altering distance function of type (S). Let $P, Q, f, g: M \rightarrow M$ be maps such that

(2.1.1) $F_{P x, Q y}(\varphi(s)) \geq F_{f x, g y}\left(\varphi\left(\frac{s}{c}\right)\right)$ for all $x, y \in M$ and for $s>0$ and $0<c<1$.

(2.1.2) $P(M) \subseteq g(M), Q(M) \subseteq f(M)$,

(2.1.3) either $f(M)$ or $g(M)$ is complete,

(2.1.4) the pairs $(f, P)$ and $(g, Q)$ are weakly compatible.

Then $f, g, P$ and $Q$ have a unique common fixed point in $M$.

Proof. Let $x_{0} \in M$.

Since $P(M) \subseteq g(M)$, there exists $x_{1} \in M$ such that $y_{1}=g x_{1}=P x_{0}$.

Since $Q(M) \subseteq f(M)$, there exists $x_{2} \in M$ such that $y_{2}=f x_{2}=Q x_{1}$.

Continuing in this way, we get sequences $\left\{x_{n}\right\}$ and $\left\{y_{n}\right\}$ in $M$ such that $y_{2 n+1}=g x_{2 n+1}=$ $P x_{2 n}$ and $y_{2 n+2}=f x_{2 n+2}=Q x_{2 n+1}, n=0,1,2 \cdots$

Since $\varphi$ is continuous at 0 and vanishes only at 0 , it follows that for given $s>0$ there exists $r>0$ such that $\frac{s}{2}>\varphi(r)$. Now

$$
\begin{aligned}
F_{y_{2 n+1}, y_{2 n+2}}(s) & \geq F_{y_{2 n+1}}, y_{2 n+2}(\varphi(r)) \\
& =F_{P x_{2 n}}, Q x_{2 n+1}(\varphi(r)) \\
& \geq F_{f x_{2 n}}, g x_{2 n+1}\left(\varphi\left(\frac{r}{c}\right)\right) \\
& =F_{y_{2 n}, y_{2 n+1}}\left(\varphi\left(\frac{r}{c}\right)\right)
\end{aligned}
$$

Similarly,

$$
F_{y_{2 n+1}, y_{2 n}}(s) \geq F_{y_{2 n}, y_{2 n-1}}\left(\varphi\left(\frac{r}{c}\right)\right) .
$$

Thus

$$
F_{y_{n+1}, y_{n}}(s) \geq F_{y_{n}, y_{n-1}}\left(\varphi\left(\frac{r}{c}\right)\right) \geq \cdots \geq F_{y_{1}, y_{0}}\left(\varphi\left(\frac{r}{c^{n}}\right)\right) .
$$

Hence from Lemma $1.15,\left\{y_{n}\right\}$ is Cauchy.

Suppose $g(M)$ is complete.

Then there exist $z, v \in M$ such that $y_{2 n+1}=g x_{2 n+1} \rightarrow z=g v$.

Since $\left\{y_{n}\right\}$ is Cauchy, we have $y_{n} \rightarrow z$.

Again for given $s>0$ there exists $r>0$ such that $\frac{s}{2}>\varphi(r)$. Now,

$$
\begin{aligned}
F_{z, Q v}(s) & \geq t\left(F_{z, y_{2 n+1}}(\varphi(r)), F_{y_{2 n+1}, Q v}(s-\varphi(r))\right), \\
& \geq t\left(F_{z, y_{2 n+1}}(\varphi(r)), F_{P x_{2 n}, Q v}(\varphi(r))\right), \\
& \geq t\left(F_{z, y_{2 n+1}}(\varphi(r)), F_{f x_{2 n}, g v}\left(\varphi\left(\frac{r}{c}\right)\right)\right), \quad \text { from } \\
& =t\left(F_{z}, y_{2 n+1}(\varphi(r)), F_{y_{2 n}, g v}\left(\varphi\left(\frac{r}{c}\right)\right)\right), \\
& \rightarrow 1 \text { as } n \rightarrow \infty .
\end{aligned}
$$

since $y_{n} \rightarrow z$ and $t$ is a continuous Hadzic type $t$-norm .

Thus $z=Q v$. Hence

$$
g v=z=Q v .
$$

Since $z=Q v \in Q(M) \subseteq f(M)$, there exists $u \in M$ such that

$$
z=f u \text {. }
$$

Now

$$
F_{P u, z}(\varphi(s))=F_{P u, Q v}(\varphi(s)) \geq F_{f u, g v}\left(\varphi\left(\frac{s}{c}\right)\right)=F_{z, z}\left(\varphi\left(\frac{s}{c}\right)\right)=1 .
$$

Thus $P u=z$. Hence

$$
P u=z=f u \text {. }
$$


Since $(f, P)$ is weakly compatible and from (2.3), we have $P z=f z$. Now from (2.1.1), we have

$F_{P z, z}(\varphi(s))=F_{P z, Q v}(\varphi(s)) \geq F_{f z, g v}\left(\varphi\left(\frac{s}{c}\right)\right)=F_{P z, z}\left(\varphi\left(\frac{s}{c}\right)\right)$

$$
\geq F_{P z, z}\left(\varphi\left(\frac{s}{c^{2}}\right)\right) \cdots \geq F_{P z, z}\left(\varphi\left(\frac{s}{c^{n}}\right)\right) \rightarrow 1 \quad \text { as } \quad n \rightarrow \infty .
$$

Thus $P z=z$. Hence

$$
z=P z=f z .
$$

Since $(g, Q)$ is weakly compatible, from $(2.1)$, we have $g z=Q z$.

From (2.1.1), we have

$F_{z, Q z}(\varphi(s))=F_{P u, Q z}(\varphi(s)) \geq F_{f u, g z}\left(\varphi\left(\frac{s}{c}\right)\right)=F_{z, Q z}\left(\varphi\left(\frac{s}{c}\right)\right)$

$$
\geq F_{z, Q z}\left(\varphi\left(\frac{s}{c^{2}}\right)\right) \cdots \geq F_{z, Q z}\left(\varphi\left(\frac{s}{c^{n}}\right)\right) \rightarrow 1 \quad \text { as } \quad n \rightarrow \infty .
$$

Thus $Q z=z$. Hence

$$
z=Q z=g z .
$$

From (2.4) and (2.5), $z$ is a common fixed point of $P, Q, f$ and $g$. Suppose $z^{\prime}$ is another common fixed point of $P, Q, f$ and $g$. Then From (2.1.1), we have

$$
\begin{array}{r}
F_{z, z^{\prime}}(\varphi(s))=F_{P z, Q z^{\prime}}(\varphi(s)) \geq F_{f z, g z^{\prime}}\left(\varphi\left(\frac{s}{c}\right)\right)=F_{z, z^{\prime}}\left(\varphi\left(\frac{s}{c}\right)\right) \\
\geq F_{z, z^{\prime}}\left(\varphi\left(\frac{s}{c^{2}}\right)\right) \cdots \geq F_{z, z^{\prime}}\left(\varphi\left(\frac{s}{c^{n}}\right)\right) \rightarrow 1 \quad \text { as } \quad n \rightarrow \infty .
\end{array}
$$

Thus $z^{\prime}=z$. Hence $z$ is the unique common fixed point of $P, Q, f$ and $g$. Similarly the theorem holds when $f(M)$ is complete.

Recently, Sastry et.al. [8] proved the following theorem for a multivalued map in a complete Menger space with Hadzic type $t$-norm.

2.2. Theorem. ([8]). Let $(M, F, t)$ be a complete Menger space with a continuous Hadzic type $t$ - norm ' $t$ ' , $\varphi$ be an altering distance function of type (S) and $P$ be a multivalued map of $M$ into the class of nonempty subsets of $M$. Suppose that there exists $0<c<1$ such that for any $x, y \in M, F_{u, v}(\varphi(s)) \geq F_{x, y}\left(\varphi\left(\frac{s}{c}\right)\right)$ for all $s>0$, whenever $u \in P x$, $v \in P y$.

Then $P$ has a unique fixed point $z \in M$ and $P z=\{z\}$.

Now we extend this theorem for two pairs of hybrid mappings.

2.3. Definition. Let $(M, F, t)$ be a Menger space and $f: M \rightarrow M, P$ be a multi valued map of $M$ into the class of nonempty subsets of $M$. Then $f$ is said to be $P$ - weakly commuting at $x \in M$ if $f^{2} x \in P f x$.

2.4. Theorem. Let $(M, F, t)$ be a Menger space with a continuous Hadzic type $t$ - norm ' $t$ ' and $\varphi$ be an altering distance function of type $(\mathrm{S})$. Let $P$ and $Q$ be multivalued maps of $M$ into the class of nonempty subsets of $M$ and $f$ and $g$ be self maps on $M$. Suppose that there exists $0<c<1$ such that for any $x, y \in M$,

(2.4.1) $F_{u, v}(\varphi(s)) \geq F_{f x, g y}\left(\varphi\left(\frac{s}{c}\right)\right)$ for all $s>0$, whenever $u \in P x, v \in Q y$.

(2.4.2) $P(M) \subseteq g(M), \quad Q(M) \subseteq f(M)$,

(2.4.3) either $f(M)$ or $g(M)$ is complete,

(2.4.4) $f$ is $P$-weakly commuting and $g$ is $Q$-weakly commuting at their coincidence points.

Then the pairs $(f, P)$ and $(g, Q)$ have a common coincidence point in $M$. 
Proof. Let $x_{0} \in M$.

Since $P\left(x_{0}\right) \subseteq g(M)$, there exists $x_{1} \in M$ such that $y_{1}=g x_{1} \in P x_{0}$.

Since $Q\left(x_{1}\right) \subseteq f(M)$, there exists $x_{2} \in M$ such that $y_{2}=f x_{2} \in Q x_{1}$.

Continuing in this way, we get sequences $\left\{x_{n}\right\}$ and $\left\{y_{n}\right\}$ in $M$ such that $y_{2 n+1}=g x_{2 n+1} \in$ $P x_{2 n}$ and $y_{2 n+2}=f x_{2 n+2} \in Q x_{2 n+1}, n=0,1,2 \cdots$

$$
\begin{aligned}
F_{y_{2 n+1}, y_{2 n+2}}(\varphi(s)) & \geq F_{f x_{2 n}, g x_{2 n+1}}\left(\varphi\left(\frac{s}{c}\right)\right), \\
& =F_{y_{2 n}, y_{2 n+1}}\left(\varphi\left(\frac{s}{c}\right)\right)
\end{aligned}
$$

Similarly,

$$
F_{y_{2 n}, y_{2 n+1}}(\varphi(s)) \geq F_{y_{2 n-1}, y_{2 n}}\left(\varphi\left(\frac{s}{c}\right)\right) .
$$

Thus

$$
F_{y_{n}, y_{n+1}}(\varphi(s)) \geq F_{y_{n-1}, y_{n}}\left(\varphi\left(\frac{s}{c}\right)\right)
$$

Since $\varphi$ is continuous at 0 and vanishes only at 0 , it follows that for given $s>0$ there exists $r>0$ such that $\frac{s}{2}>\varphi(r)$. Now

$$
F_{y_{n}, y_{n+1}}(s) \geq F_{y_{n}, y_{n+1}}(\varphi(r)) \geq F_{y_{n-1}, y_{n}}\left(\varphi\left(\frac{r}{c}\right)\right) \geq \cdots \geq F_{y_{0}, y_{1}}\left(\varphi\left(\frac{r}{c^{n}}\right)\right) .
$$

Hence from Lemma 1.15, $\left\{y_{n}\right\}$ is Cauchy sequence in $M$.

Suppose $f(M)$ is complete.

Then there exist $z, p \in M$ such that $y_{n} \rightarrow z=f p$.

Let $z_{1} \in P p$. Since $y_{2 n+2}=f x_{2 n+2} \in Q x_{2 n+1}$, from (2.4.1), we have

$$
\begin{aligned}
F_{f p, z_{1}}(s) & \geq t\left(F_{f p, f x_{2 n+2}}(\varphi(r)), F_{f x_{2 n+2}, z_{1}}(s-\varphi(r))\right) \\
& \geq t\left(F_{z, y_{2 n+2}}(\varphi(r)), F_{f x_{2 n+2}, z_{1}}(\varphi(r))\right) \\
& \geq t\left(F_{z, y_{2 n+2}}(\varphi(r)), F_{f p, g x_{2 n+1}}\left(\varphi\left(\frac{r}{c}\right)\right)\right) \\
& =t\left(F_{z, y_{2 n+2}}(\varphi(r)), F_{z, y_{2 n+1}}\left(\varphi\left(\frac{r}{c}\right)\right)\right) \\
& \rightarrow 1 \text { as } n \rightarrow \infty .
\end{aligned}
$$

since $y_{n} \rightarrow z$ and $t$ is a continuous Hadzic type $t$-norm .

Thus $F_{f p, z_{1}}(s)=1$ for $s>0$ so that $f p=z_{1}$. Thus

$$
f p \in P p \text {. }
$$

Since $z=f p \in P p \subseteq g(M)$, there exists $q \in M$ such that $z=f p=g q$.

Let $z_{2} \in Q q$. Since $y_{2 n+1}=g x_{2 n+1} \in P x_{2 n}$, from (2.4.1), we have

$$
\begin{aligned}
F_{g q, z_{2}}(s) & \geq t\left(F_{g q, g x_{2 n+1}}(\varphi(r)), F_{g x_{2 n+1}}, z_{2}(s-\varphi(r))\right), \\
& \geq t\left(F_{z, y_{2 n+1}}(\varphi(r)), F_{g x_{2 n+1}, z_{2}}(\varphi(r))\right), \\
& \geq t\left(F_{z, y_{2 n+1}}(\varphi(r)), F_{f x_{2 n}, g q}\left(\varphi\left(\frac{r}{c}\right)\right)\right), \\
& =t\left(F_{z, y_{2 n+1}}(\varphi(r)), F_{y_{2 n}, z}\left(\varphi\left(\frac{r}{c}\right)\right)\right), \\
& \rightarrow 1 \text { as } n \rightarrow \infty .
\end{aligned}
$$

since $y_{n} \rightarrow z$ and $t$ is a continuous Hadzic type $t$-norm .

Thus $F_{g q, z_{2}}(s)=1$ for $s>0$ so that $g q=z_{2}$. Thus

$$
g q \in Q q \text {. }
$$

From (2.6) and (2.7), $p$ is a coincidence point of $f$ and $P ; q$ is a concidence point of $g$ and $Q$.

From (2.4.4), $f z \in P z$ and $g z \in Q z$. Thus $z$ is a common coincidence point of the hybrid pairs $(f, P)$ and $(g, Q)$.

Acknowledgement . The authors are thankful to the referee for his valuable suggestions. 


\section{References}

[1] Choudhury, B.S. and Das, Krishnapada. A new contraction principle in Menger spaces , Acta Mathematica Sinica, English Series, 24(8),(2008), 1379-1386.

[2] Choudhury,B.S.,Das,K. and Dutta,P.N.,A fixed point result in Menger spaces using a real function, Acta Math.Hungarica,122(3),(2009),203-216.

[3] Dutta,P.N.,Choudhury,B.S. and Das,K.,Some fixed point results in Menger spaces using a control function, Surveys in Mathematics and its applications,4,(2009),41-52.

[4] Hadzic, O. On $(\epsilon-\lambda)$ - topology in probabilistic locally convex spaces, Glasnik Matem 13 (33), (1978), $193-297$.

[5] Jungck,G and Rhoades,B.E. , Fixed points for set valued functions without continuity condition , Indian.J.Pure.Appl.Math., 29(3),(1998),227-238.

[6] Menger, K. Statistical metrics, Proc.of the National Academy of Sciences of the United States of America , 28,(1942),535-537.

[7] Mihet,D,Altering distances in probabilistic Menger spaces, Nonlinear Anal.,71,(2009),27342738 .

[8] Sastry, K.P.R., Babu, G.V.R. and Sandhya, M.L. Fixed point theorems in Menger spaces for a contractive map under the influence of an altering distace function of type $(\mathrm{S})$ (Communicated).

[9] Schweizer, B. and Sklar, A. Statistical metric spaces, Pacific J.Math., 10 (1960), 313 - 334.

[10] Sehgal, V.M. and Bharucha - Reid, A.T. Fixed points of contraction mappings on probabilistic metric spaces, Math. Systems theory, 6 (1972), 97 - 102. 
